\title{
A comprehensive approach to classify reactive power consumption in transmission technologies
}

\author{
S. Höhn, A. Semerow and M. Luther \\ Chair of Electrical Energy Systems \\ University of Erlangen-Nuremberg \\ Konrad-Zuse-Straße 3-5 - 91052 Erlangen (Germany) \\ Phone number: +49 91318523453
}

E-mail: sebastian.hoehn@ees.fau.de, anatoli.semerow@ees.fau.de, matthias.luther@ees.fau.de

\begin{abstract}
Reactive power consumption is an essential feature of any electrical equipment applied in electrical power systems. To accomplish its main task, i.e. the transfer of active power, an electrical device interacts with the system it is connected to. A common aim is to reduce the reactive power flow on the terminals of the equipment to a minimum. Therefore it's necessary to compensate the reactive power consumption by means of adequate measures. This can't be reached without precise investigation on the sources of reactive power emergence. This paper investigates the physical phenomena underlying the reactive power behavior of HVAC and HVDC transmission technologies. After having introduced a common mathematical definition of active, apparent and reactive power, reactive power is categorized and the concept is applied to analyze the aforementioned technologies.
\end{abstract}

\section{Key words}

HVDC, HVAC, reactive power, power quality, converter technology

\section{Introduction}

The increasing infeed of electrical energy obtained through renewable energy sources like wind, water and sun leads to the progressing geographical decoupling of generation and load [1]. Where fossil power plants are built in close vicinity to heavy load centers, the site of wind turbines, hydroelectric and solar (thermal) power stations is defined by natural location factors [1]. As it is crucial for achieving the changeover to a renewable driven energy system, a growing demand for the transmission of electric energy over long distances is arising worldwide. However, there are different technologies to achieve these purposes. For a specific task of transmission it's not obvious which of these potential possibilities is the most suitable.

Amongst others, reactive power consumption is a criterion to handle the comparison of transmission technologies. Reactive power has been a subject of scientific controversy for several decades [2]. As a contribution to this subject, this paper uses a common mathematical classification [3] to assemble all physical phenomena, which contribute to the reactive power consumption of the three available technologies for bulk power transmission. In contrast to other publications, where phase difference is assumed to be the only root for reactive power [4], a holistic approach towards reactive power demand of transmission technologies is conducted in this paper. A general investigation on the functional chains concerning reactive power of the involved electrical equipment gives a fine hint to take appropriate steps in the process of achieving balanced reactive power consumption.

\section{Definition and classification of reactive power}

Starting from the introduction of the terms active, apparent and reactive power, different classes of reactive power are derived from the common mathematic definition to lay the foundations for the analyses on the transmission technologies.

\section{A. Active, apparent and reactive power}

Active power is time-dependent without loss of generality. It's defined as the derivative with respect of time of electric energy, which is the product of voltage and current:

$$
\frac{\mathrm{d} W_{\text {electric }}}{\mathrm{d} \omega t}=p(\omega t)=u(\omega t) \cdot i(\omega t)
$$

It is characteristic for symmetric three-phase AC systems, that $p(\omega t)$ is constant.

For periodic systems active power $P$ is the mean value of time-dependent active power $p(\omega t)$ :

$$
P=\frac{1}{2 \pi} \int_{t_{0}}^{t_{0}+2 \pi} p(\omega t) \mathrm{d} \omega t
$$

Apparent power of a periodic system is obtained by multiplying the RMS values of voltage and current [3]:

$$
\begin{aligned}
S & =U_{\text {RMS }} \cdot I_{\text {RMS }}= \\
& =\sqrt{\frac{1}{2 \pi} \int_{t_{0}}^{t_{0}+2 \pi} u(\omega t)^{2} \mathrm{~d} \omega \mathrm{t}} \cdot \sqrt{\frac{1}{2 \pi} \int_{t_{0}}^{t_{0}+2 \pi} i(\omega t)^{2} \mathrm{~d} \omega \mathrm{t}}
\end{aligned}
$$

The physical analogy of the mathematic definition describes apparent power as the maximum effective power that could be transferred for a defined utilization of electrical equipment [3]. The relation between active and apparent power is given by the power factor $\lambda$ :

$$
\lambda=\frac{P}{S}
$$


All effects that reduce this power factor are embraced by the term of reactive power. Hence, it is determined as follows:

$$
Q=\sqrt{S^{2}-P^{2}}
$$

While reactive power is often simplified to the consequence of a phase difference between current and voltage, figure 1 shows all four categories, as they describe a separate physical phenomenon and possess an own mathematical derivation. These classes of reactive power are introduced in the next section.

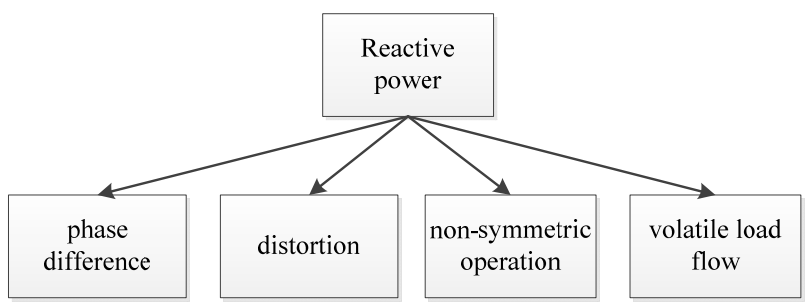

Figure 1: categorization of reactive power [3]

\section{B. Reactive power due to phase difference}

By the reason of its outstanding relevance on interconnected systems is this class of reactive power often equated with being reactive power itself. Electrical equipment, load and generation are analyzed regarding their influence on the phase difference. The balance of reactive power locally affects the voltage level of a grid node and globally maintains voltage stability of a power system.

The sinusoidal signals of voltage and current are given:

$$
u(\omega t)=\hat{U} \cos \left(\omega t+\varphi_{u}\right), i(\omega t)=\hat{I} \cos \left(\omega t+\varphi_{i}\right)
$$

The instantaneous power according to (1) is:

$$
\begin{aligned}
& p(\omega t)=\frac{1}{2} \hat{U}\left(\mathrm{e}^{\mathrm{j}\left(\omega t+\varphi_{u}\right)}+\mathrm{e}^{-\mathrm{j}\left(\omega t+\varphi_{u}\right)}\right) \cdot \frac{1}{2} \hat{I}\left(\mathrm{e}^{\mathrm{j}\left(\omega t+\varphi_{i}\right)}+\mathrm{e}^{-\mathrm{j}\left(\omega t+\varphi_{i}\right)}\right) \\
& p(\omega t)=\frac{1}{2} \hat{U} \hat{I}\left(\cos \left(2 \omega t+\varphi_{u}+\varphi_{i}\right)+\cos \left(\varphi_{u}-\varphi_{i}\right)\right)
\end{aligned}
$$

The active power as the mean value over a period is:

$$
\begin{aligned}
& P=\frac{1}{2 \pi} \int_{0}^{2 \pi} \frac{\hat{U} \hat{I}}{2}\left(\cos \left(2 \omega t+\varphi_{u}+\varphi_{i}\right)+\cos \left(\varphi_{u}-\varphi_{i}\right)\right) \mathrm{d} \omega t \\
& P=\frac{\hat{U} \hat{I}}{4 \pi} \cdot\left[\sin \left(2 \omega t+\varphi_{u}+\varphi_{i}\right) \cdot \frac{1}{2}+\cos \left(\varphi_{u}-\varphi_{i}\right) \omega t\right]_{0}^{2 \pi} \\
& P=\frac{\hat{U} \hat{I}}{4 \pi} \cdot\left(\cos \left(\varphi_{u}-\varphi_{i}\right) \cdot 2 \pi\right)=\frac{\hat{U} \hat{I}}{2} \cdot \cos \left(\varphi_{u}-\varphi_{i}\right)
\end{aligned}
$$

The apparent power of the signal is determined by (3):

$$
\begin{aligned}
S & =\sqrt{\frac{\hat{U}^{2}}{2 \pi} \int_{0}^{2 \pi} \cos ^{2}\left(\omega t+\varphi_{u}\right) \mathrm{d} \omega t} \sqrt{\frac{\hat{I}^{2}}{2 \pi} \int_{0}^{2 \pi} \cos ^{2}\left(\omega t+\varphi_{i}\right) \mathrm{d} \omega t} \\
S & =\sqrt{\frac{\hat{U}^{2} \hat{I}^{2}}{4 \pi^{2}}\left[\frac{1}{2}\left(\omega t+\frac{1}{2} \sin \left(2 \omega t+\varphi_{u}\right)\right)\right]_{0}^{2 \pi}} \cdot \\
& \cdot \sqrt{\left[\frac{1}{2}\left(\omega t+\frac{1}{2} \sin \left(2 \omega t+\varphi_{i}\right)\right)\right]_{0}^{2 \pi}}=\sqrt{\frac{\hat{U}^{2} \hat{I}^{2}}{4 \pi^{2}} \pi \cdot \pi}=\frac{\hat{U} \hat{I}}{2}
\end{aligned}
$$

The comparison of the results for active power (8) and apparent power (9) reveals their relation with regards to the phase difference:

$$
P=S \cdot \cos \left(\varphi_{u}-\varphi_{i}\right)=S \cdot \cos (\varphi)
$$

In consequence, the reactive power due to phase difference can be stated as follows:

$$
Q=\sqrt{\frac{\hat{U}^{2} \hat{I}^{2}}{4}-\frac{\hat{U}^{2} \hat{I}^{2}}{4} \cos ^{2}(\varphi)}=\frac{\hat{U} \hat{I}}{2} \sqrt{1-\cos ^{2}(\varphi)}=S \sin (\varphi)
$$

In consideration of equations (10) and (11) the definition of the complex power $\underline{S}$ is self-evident:

$$
\underline{S}=P+\mathrm{j} Q
$$

Furthermore complex power is the product of complex voltage and the complex conjugate current:

$$
\underline{S}=\underline{U} \cdot \underline{I}^{*}
$$

\section{Reactive power due to distortion}

Individual components of electrical power systems lead to a non-linear relation between voltage and current. These effects create higher harmonics, which interfere the fundamental frequency of the time-dependent signals. Power electronic switched devices and saturated power transformers are the most frequent sources of harmonic distortion in electric power systems.

Any electric signal can be depicted through the Fourier expansion:

$$
v(\omega t)=\sum_{n=-\infty}^{+\infty} A_{n} \cos (n \omega t), n \in \mathbb{N}
$$

For the determination of active and apparent power according to equations (2) and (3) it's necessary to use the Fourier series of each signal. Voltage and current of different harmonics don't contribute to the active power:

$$
\begin{aligned}
& P_{m n}=\frac{\hat{U}_{m} \hat{I}_{n}}{2 \pi} \int_{0}^{2 \pi} \cos (m \omega t) \cdot \cos (n \omega t) \mathrm{d} \omega t \\
& P_{m n}=\frac{\hat{U}_{m} \hat{I}_{n}}{2 \pi} \cdot\left[\frac{\sin ((m-n) \omega t)}{2(m-n)}+\frac{\sin ((m+n) \omega t)}{2(m+n)}\right]_{0}^{2 \pi} \\
& P_{m n}=\frac{\hat{U}_{m} \hat{I}_{n}}{2 \pi} \cdot\left(\frac{\sin (2 \pi(m-n))}{2(m-n)}+\frac{\sin (2 \pi(m+n))}{2(m+n)}\right) \\
& P_{m n}=0 \quad \forall m \neq n, m \in \mathbb{N}
\end{aligned}
$$

However, for $m=n$ a contribution does exist:

$$
\begin{aligned}
& P_{m m}=\frac{\hat{U}_{m} \hat{I}_{m}}{2 \pi} \int_{0}^{2 \pi} \cos (m \omega t) \cdot \cos (m \omega t) \mathrm{d} \omega t= \\
& =\frac{\hat{U}_{m} \hat{I}_{m}}{2 \pi} \cdot\left[\frac{1}{2} \omega t+\frac{\sin (2 m \omega t)}{4 m}\right]_{0}^{2 \pi}=\frac{\hat{U}_{m} \hat{I}_{m}}{2 \pi} \cdot(\pi)=\frac{\hat{U}_{m} \hat{I}_{m}}{2}
\end{aligned}
$$

The apparent power for any arbitrary combination of harmonics is:

$$
\begin{aligned}
S_{m n} & =\frac{\hat{U}_{m} \hat{I}_{n}}{2 \pi} \cdot \sqrt{\int_{0}^{2 \pi} \cos ^{2}(m \omega t) \mathrm{d} \omega t} \cdot \sqrt{\int_{0}^{2 \pi} \cos ^{2}(n \omega t) \mathrm{d} \omega t} \\
S_{m n} & =\frac{\hat{U}_{m} \hat{I}_{n}}{2 \pi} \cdot \sqrt{\left[\frac{1}{2} \omega t+\frac{1}{4 m} \sin (2 m \omega t)\right]_{0}^{2 \pi}} \cdot \\
& \cdot \sqrt{\left[\frac{1}{2} \omega t+\frac{1}{4 n} \sin (2 n \omega t)\right]_{0}^{2 \pi}}=\frac{\hat{U}_{m} \hat{I}_{n}}{2 \pi} \cdot \sqrt{\pi \cdot \pi}=\frac{\hat{U}_{m} \hat{I}_{n}}{2}
\end{aligned}
$$

Thus higher harmonics of a signal have the same influence on the apparent power like the fundamental components (see equation (2)). 
As per its definition in equation (5), reactive power emerges unequivocally due to harmonic distortion.

\section{Reactive power due to volatile load-flow}

Loads usually don't have time-constant active power consumption. They rather fluctuate in a pretty slow manner. This fact influences the power factor [3], which is proved by use of a simple example shown in Figure 2.

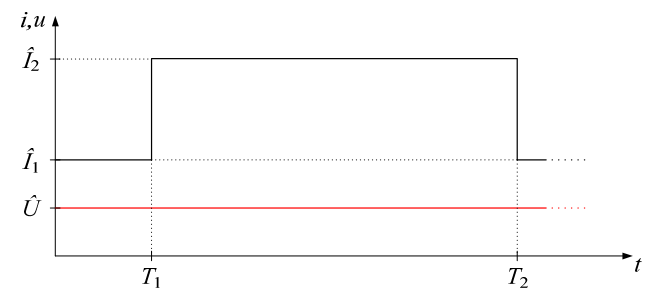

Figure 2: Fluctuation of a DC-load

Active and apparent power for the given load curve are determined in (18) and (19):

$$
\begin{aligned}
& P=\frac{1}{T_{2}} \int_{0}^{T_{2}} \hat{U} \cdot \hat{I} \mathrm{~d} t=\frac{1}{T_{2}}\left(\int_{0}^{T_{1}} \hat{U} \cdot \hat{I}_{1} \mathrm{~d} t+\int_{T_{1}}^{T_{2}} \hat{U} \cdot \hat{I}_{2} \mathrm{~d} t\right)= \\
& P=\frac{1}{T_{2}}\left(T_{1} \hat{U} \hat{I}_{1}+\left(T_{2}-T_{1}\right) \hat{U} \hat{I}_{2}\right)=\hat{U} \hat{I}_{1} \frac{T_{1}}{T_{2}}+\hat{U} \hat{I}_{2} \frac{T_{2}-T_{1}}{T_{2}} \\
& S=\frac{1}{T_{2}}\left(\sqrt{\int_{0}^{T_{2}} \hat{U}^{2} \mathrm{~d} t} \cdot \sqrt{\int_{0}^{T_{2}} \hat{I}^{2} \mathrm{~d} t}\right)= \\
& =\frac{1}{T_{2}}\left(\sqrt{\int_{0}^{T_{2}} \hat{U}^{2} \mathrm{~d} t} \cdot \sqrt{\int_{0}^{T_{1}} \hat{I}_{1}^{2} \mathrm{~d} t+\int_{T_{1}}^{T_{2}} \hat{I}_{2}^{2} \mathrm{~d} t}\right)= \\
& =\frac{\hat{U}}{T_{2}} \sqrt{T_{2}} \sqrt{\hat{I}_{1}^{2} T_{1}+\hat{I}_{2}^{2}\left(T_{2}-T_{1}\right)}=\frac{\hat{U}}{\sqrt{T_{2}}} \sqrt{\hat{I}_{1}^{2} T_{1}+\hat{I}_{2}^{2}\left(T_{2}-T_{1}\right)}
\end{aligned}
$$

The corresponding reactive power can be determined with the general equation (5).

\section{E. Reactive power due to non-symmetric operation}

In addition to the aforementioned three categories another reactive power class has to be considered for three-phase AC-systems. A three-phase system is called "symmetric" when all time-dependent phase values have identical magnitude and frequency in each phase and the phase difference between all three phases is equal to 120 degrees. Whenever electrical equipment impacts symmetry, additional reactive power is to be applied.

A single phase load shall be examined towards reactive power:

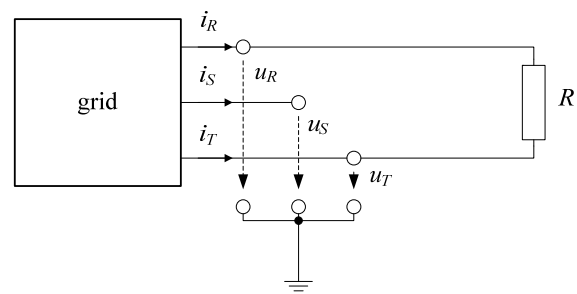

Figure 3: single-phase load

The ohmic resistance is connected between the phases $\mathrm{R}$ and $\mathrm{T}$. Therefore the active power at the load is:

$$
\begin{aligned}
& P=\frac{1}{2 \pi} \int_{0}^{2 \pi} u_{R T}(\omega t) \cdot i_{R}(\omega t) \mathrm{d} \omega t=\int_{0}^{2 \pi} \frac{\sqrt{3} \hat{U} \hat{I}}{2 \pi} \cos ^{2}\left(\omega t-\frac{\pi}{6}\right) \mathrm{d} \omega t \\
& =\frac{3 \hat{U}^{2}}{2 \pi R}\left[\frac{1}{2} \omega t+\frac{1}{4} \sin \left(2 \omega t-\frac{\pi}{3}\right)\right]_{0}^{2 \pi}=\frac{3 \hat{U}^{2}}{2 R}
\end{aligned}
$$

Apparent power of a three-phase system is defined as follows [3]:

$$
S=\sqrt{{U_{R, \mathrm{RMS}}}^{2}+{U_{S, \mathrm{RMS}}}^{2}+U_{T, \mathrm{RMS}}{ }^{2}} \cdot \sqrt{{I_{R, \mathrm{RMS}}}^{2}+{I_{S, \mathrm{RMS}}}^{2}+{I_{T, \mathrm{RMS}}}^{2}}
$$

Assuming pure sinusoidal voltages and currents it's possible to determine the apparent power through equation (9):

$$
S=\sqrt{\frac{3}{2} \hat{U}^{2} \cdot \hat{I}_{R}^{2}}=\frac{3 \hat{U}^{2}}{\sqrt{2} R}
$$

The power factor of the examined non-symmetric operation is:

$$
\lambda=\frac{P}{S}=\frac{1}{\sqrt{2}} \approx 0.707
$$

\section{Reactive power consumption transmission technologies}

After having introduced the specific reactive power phenomena, this chapter aims at investigating their influence on HVDC and HVAC transmission technologies. AC-connections basically comprise transmission line and two power transformers at both ends of the line. The behavior of a HVDC application regarding reactive power consumption is largely identical to the behavior of the converter stations. This paper addresses both topologies, classic HVDC with line commutated converters using thyristor valves (LCC) and HVDC with voltage sourced converters (VSC) using IGBT modules.

To simplify the comprehension of the following chapters, a 6-pulse converter bridge is depicted in figure 4 .

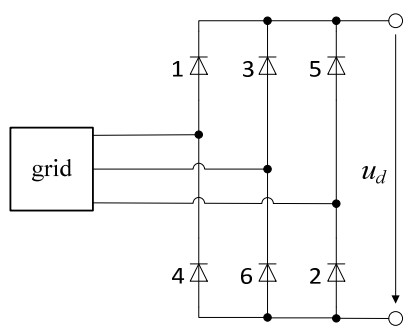

Figure 4: converter with diode valves

\section{A. Phase difference on HVAC transmission}

To analyze the effect of a three-phase AC-transmission line, figure 5 shows the equivalent circuit of the positive sequence with an ohmic load $R_{(1)}$ at its end.

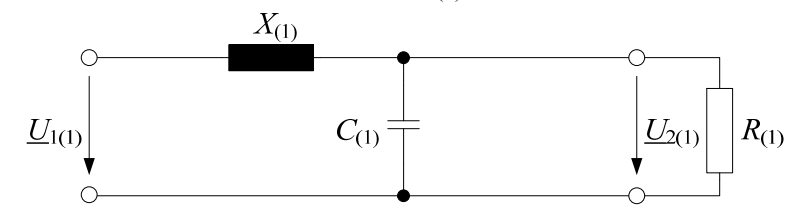

Figure 5: Equivalent positive sequence circuit of a transmission line 
As it contains a capacitive and an inductive component it's obvious, that there won't be any phase difference if inductance and capacity would neutralize each other. As reactive power is the imaginary part of complex power $\underline{S}$ the reactive power consumption of these two components can be stated:

$$
\begin{aligned}
& Q_{C}=\operatorname{Im}\left\{\underline{U}_{C} \underline{I}_{C}^{*}\right\}=\operatorname{Im}\left\{-\underline{U}_{C} \cdot \mathrm{j} \omega C_{(1)} \cdot \underline{U}_{C}^{*}\right\}=-\omega C_{(1)} U_{C}{ }^{2} \\
& Q_{L}=\operatorname{Im}\left\{\underline{U}_{L} \cdot \underline{I}_{L}^{*}\right\}=\operatorname{Im}\left\{\mathrm{j} \omega L_{(1)} \underline{I}_{L} \cdot \underline{I}_{L}{ }^{*}\right\}=\omega L_{(1)} I_{L}{ }^{2}
\end{aligned}
$$

$Q_{C}$ depends on the voltage magnitude, which remains within a well-defined tolerance range for all undisturbed operating points. $Q_{L}$, however, varies in dependency on the transmitted power. The summation of the two factors reveals an operating point, where the line neither produces nor absorbs reactive power.

$$
Q_{C} \stackrel{!}{=} Q_{L} \text { or } \operatorname{Im}\left\{X_{(1)}+C_{(1)} \| R_{(1)}\right\} \stackrel{!}{=} 0
$$

When equation (26) is satisfied, the line is operated with "natural power" $P_{\text {nat. }}$. Figure 6 shows the reactive power consumption of a $380 \mathrm{kV}$ transmission line. Its typical perunit-length parameters were adopted from [5].

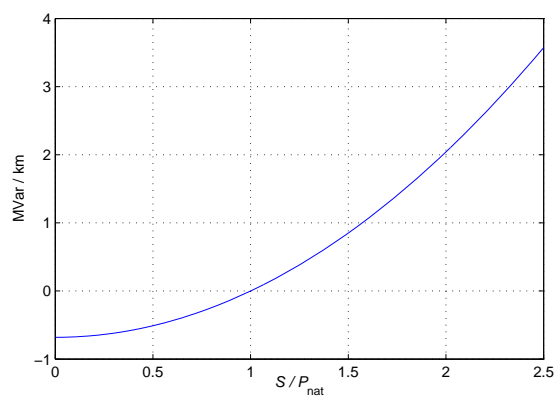

Figure 6: reactive power consumption of a transmission line

It's obvious, that the transmission line consumes reactive power when operated above natural power and has a capacitive behavior when charged lower than the natural load. The task of compensation strategies is to operate the transmission line as close as possible to its natural load. That minimizes the reactive power production or consumption, respectively. To reach this target for a wide range of operating points, reactive-power compensation devices control the surge impedance by switching capacitive and inductive elements. The use of power electronics has accelerated the controller velocity and has provided continuous step control.

\section{B. Phase difference on HVDC transmission}

As there are different processes influencing reactive power performance on LCC and VSC technology, they are discussed separately.

Two distinct phenomena result in a phase delay of the DCcurrent and thus contribute to the inductive behavior of rectifier and inverter. To expose their basic schemes, the influences of commutation and converter control are isolated from other effects and examined in highly simplified examples.

For the examination of commutation these simplifications are a rectangular phase currents and sinusoidal phase-tophase voltages. The resulting current of phase $\mathrm{S}$ under neglect of commutation (left) and for an angle of overlap $\beta$ equal to $30^{\circ}$ (right) are depicted in figure 7 .
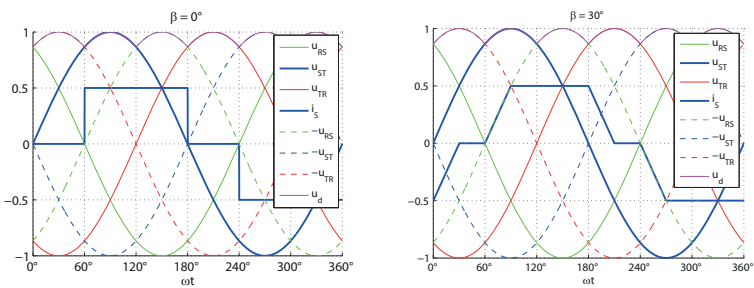

Figure 7: influence of commutation on the phase difference

It can be easily derived from the trend of the phase current, that the phase difference due to commutation $\Delta \varphi_{\text {Kom }}$ is $\beta / 2$. As $\beta$ is a function of the firing angle and the short-circuit impedance there are rather small chances to counteract the origin of the phase difference due to commutation.

The controllability of a classic HVDC interconnection by adjusting the fire impulses of the thyristor valves is paid by the disadvantage of increasing the reactive power consumption. The longer the impulses are delayed, the higher gets the phase difference in the rectifier mode. Considering the inverter, however, the higher the extinction angle gets, the higher is the reactive power consumption. These phenomena are illustrated in figure 8 .
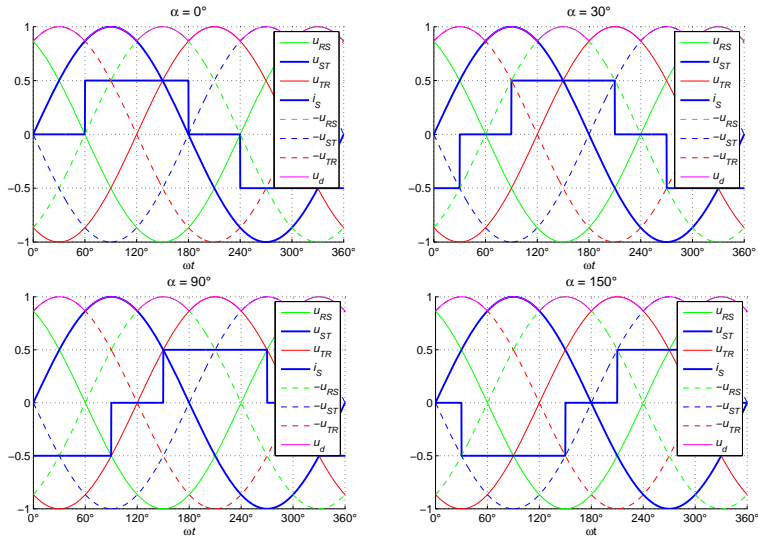

Figure 8: influence of fire impulse control on phase difference

The impact of the additional phase difference due to the control activity on the reactive power consumption can be stated in dependency of fundamental frequency apparent power $\underline{S}_{1}$ :

$$
Q_{\text {Control }}=\operatorname{Im}\left\{\underline{S}_{1}\right\}=U_{1, \text { eff }} \cdot I_{1, \text { eff }} \sin (\alpha)
$$

The locus of reactive power consumption due to controllability, as shown in figure 9, comprises active and reactive power as a function of the firing angle $\alpha[6]$. The converter has an inductive behavior in all operating points. Involving the tap changers of the converter transformers as control variables gives rise to a degree of freedom, which can be used to optimize the HVDC control towards reactive power consumption. 


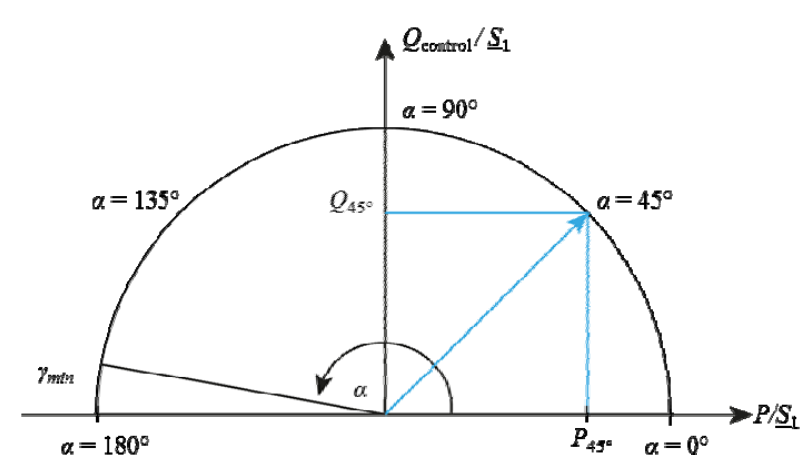

Figure 9: P-/Q-locus of a classic HVDC scheme [6]

For VSC topology, the rectifier ordinarily works as an uncontrolled diode bridge, whereas the inverter can set magnitude and phase of the output voltage independently by means of space vector modulation. Thus, the inverter can absorb or inject reactive power. The range of operation is provided by a manufacturer (figure 10).

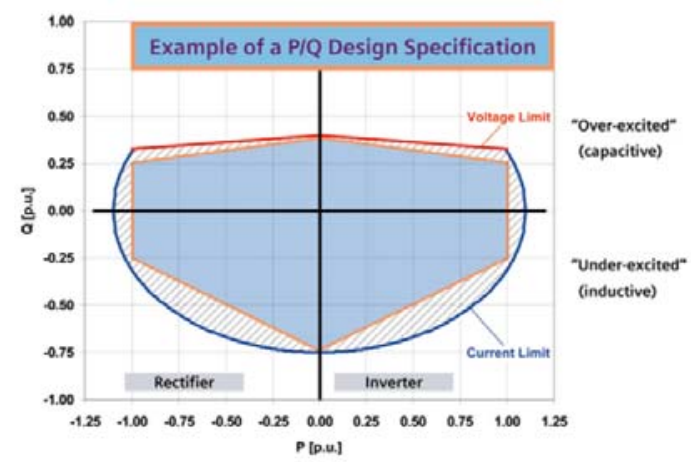

Figure 10: P-/Q-controllability of a VSC HVDC system, adopted from [7]

There are some additional topics that are not addressed in this paper. For instance the transformers as ohmicinductive components and the influences of a possible DCinductor contribute to the overall phase delay of the current.

\section{Distortion on HVAC lines}

For distortion analyses, AC transmission lines could be rather treated as passive elements that don't create higher harmonics. However, a line transfers signal harmonics and thus has impact on the signal spectrum. The complex propagation constant $\gamma$ comprises of the attenuation constant $\alpha$ and the phase constant $\beta$.

$$
\underline{\gamma}=\alpha+\mathrm{j} \beta=\sqrt{\left(R^{\prime}+\mathrm{j} \omega L^{\prime}\right) \cdot\left(G^{\prime}+\mathrm{j} \omega \mathrm{C}^{\prime}\right)}
$$

Both constants are in general frequency-dependent. For typical values of a $380 \mathrm{kV}$ transmission line, attenuation and phase difference are depicted in figure 11 . The fundamental frequency and very low harmonics up to the fifth are less damped than higher harmonics, where a constant behavior can be stated. The phase constant is a linear function of frequency. In conclusion, the observations for harmonics higher than the fifth are consistent with the Heaviside condition of a distortion-free line [8].

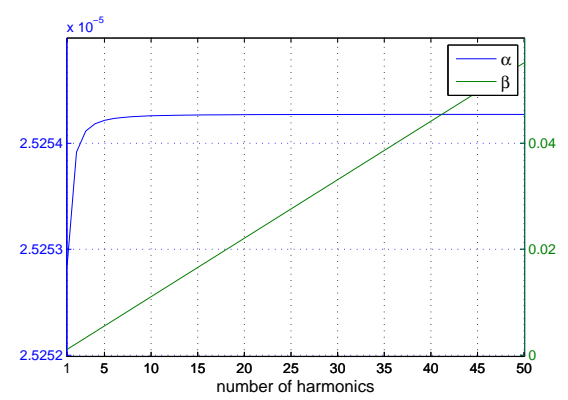

Figure 11: attenutation and phase constant of a $380 \mathrm{kV}$ line

Another point, which influences the harmonic spectrum of a transmission signal, is the non-linear hysteresis of the ferrite core of a power transformer. The higher the utilization, the greater is the resulting distortion.

\section{Distortion on HVDC systems}

Unlike the HVAC transmission, the HVDC technology is a serious source of distortion. Different origins contribute to the overall behavior. They are discussed in the chapter. Assuming a large inductor on the DC-side and a small short circuit inductivity, the phase currents can be considered to be rectangular. The Fourier analysis reveals a spectrum of a $p$-pulse converter with its characteristic harmonics $n$ given in equation (30) [6]:

$$
n=k \cdot p \pm 1, k \in \mathbb{N}_{0}
$$

In order to expose the influence of commutation on the phase current distortion, a trapezoidal signal with a linear commutation is examined regarding its harmonics. The harmonics up to the $40^{\text {th }}$ are plotted in figure 12. It's evident, that the commutation reduces the harmonic distortion of the phase current.
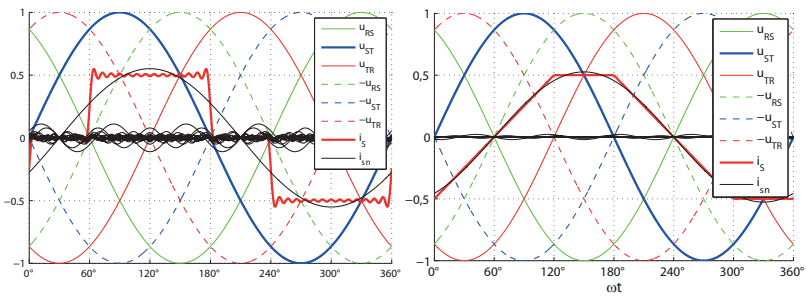

Figure 12: influence of commutation on phase current harmonics

Filtering or control with regards to elimination of certain harmonics can compensate the reactive power consumption due to distortion of the phase current.

As the DC-voltage is not a pure DC-signal, the voltage ripple of a classic HVDC connection is another principle contributing to the overall reactive power consumption. Using the equivalent circuits for commutation and two conducting valve mode [5] results in the equation for the corresponding DC-voltages $U_{\mathrm{DC}, \mathrm{Kom}}$ and $U_{\mathrm{DC}, 2 \mathrm{Vv}}$.

$$
\begin{aligned}
& U_{\mathrm{DC}, \mathrm{Kom}}=\frac{3}{2} \hat{U}_{p} \cos \left(\omega t+\varphi_{\mathrm{Kom}}\right) \\
& U_{\mathrm{DC}, 2 \mathrm{VV}}=\sqrt{3} \hat{U}_{p} \cos \left(\omega t+\varphi_{2 \mathrm{Vv}}\right)
\end{aligned}
$$

In equation (30) $\hat{U} p$ is the magnitude of the voltage source, the phase angles are given in table 1 . 
Table 1: angles for valve participations of a 6-pulse converter

\begin{tabular}{|c|c|c|c|c|c|c|}
\hline valve participation & 123 & 234 & 345 & 456 & 561 & 612 \\
\hline$\varphi_{\text {Kom }}$ & $-\pi / 3$ & $-2 \pi / 3$ & $-\pi$ & $2 \pi / 3$ & $\pi / 3$ & 0 \\
\hline valve participation & 12 & 23 & 34 & 45 & 56 & 61 \\
\hline$\varphi_{2 \mathrm{Vv}}$ & $-\pi / 6$ & $-\pi / 2$ & $-5 \pi / 6$ & $5 \pi / 6$ & $\pi / 2$ & $\pi / 6$ \\
\hline
\end{tabular}

The voltage drop due to commutation has impacts on the mean value and the ripple of the DC-voltage. There are two factors influencing the severity of the impact: The firing angle $\alpha$ and the angle of overlap $\beta . U_{\mathrm{DC}}$ for an entire period is shown in figure 13 .
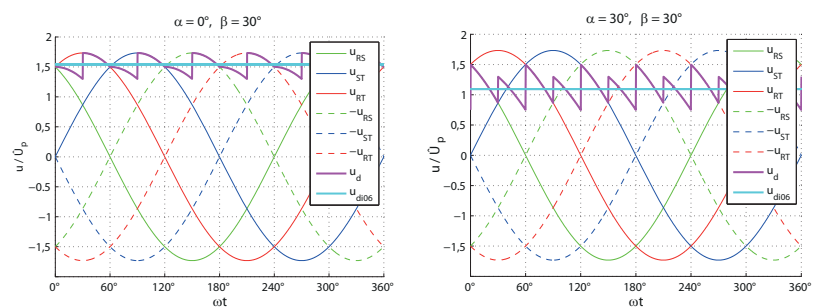

Figure 13: influence of commutation on DC-voltage ripple

Once more, the results show, that also regarding the distortion on the DC side, the operating point should be as close as possible to full power operation at a rectifier angle close to zero and a minimal extinction angle at the inverter.

With the voltage not being a pure DC-signal anymore, the inductive and capacitive elements of the transmission line can't be neglected further on.

The VSC HVDC can significantly reduce the distortion on AC-terminals and DC-circuit by using several discrete steps to form the AC-voltages at the inverter. This "multilevel" approach is state-of-the-art for VSC converters in power systems [9].

\section{E. Volatile load-flow}

Originally, the definition of reactive power due to volatile load-flow aimed at covering the effect of fluctuating load, which often, especially in industrial applications, follow a slow, cyclic power consumption. For a renewable driven power system, this definition must be extended to a volatile energy generation, which is characteristic for wind and solar based infeed. In comparison to the analysis of fluctuating load of a DC-system, performed in chapter 2, a similar approach did not show any significant differences. These elementary calculations should be extended to real configurations to estimate the outcome of this aspect.

\section{F. Non-symmetric operation}

For any electrical device it's impossible to construct a total symmetric design with identical distances between phases and equidistance to earth at the same time. Therefore a complete symmetry cannot be reached. However, it can be reduced to a tolerable level.

The transposition of phases is a well-known measure to compensate non-symmetric configuration of transmission lines bridging long distances. Adequate transposition schemes are described in literature [5], [8].

As mentioned hereinbefore, a converter has two different operating states, commutation and two conducting valves mode. For a DC-periodic view on the behavior, both of them seem to be non-symmetric states. For instance, while conducting of valves 1 and 2 , the system arrangement is abstractly seen similar to a load between phases $\mathrm{R}$ and $\mathrm{T}$ according to figure 3. However, the defining rules for symmetry are obtained for a whole ACperiod. A $120^{\circ}$ phase difference between currents and voltages as well as an identical magnitude of all phase values is kept for equidistant firing pulses.

It's evident, that this prerequisite can't be valid for practical operation. As there are fluctuations and oscillations, the control has to adjust firing and extinction angels of the rectifier and the inverter permanently to maintain a steady transmission. Therefore a converter requires additional reactive power in consequence of non-equidistant firing impulses.

\section{Conclusion}

A comprehensive approach to classify reactive power consumption of different transmission technologies was performed. The emphasis within the comparison was put on HVAC and classical HVDC concepts. Nevertheless, wherever the VSC HVDC displayed different behavior, it was highlighted separately.

Based on the mathematical definition, reactive power is categorized into four main classes, whereas lots of works are only considering reactive power behavior regarding influences on the phase difference. This approach is the basis for a holistic consideration of all physical phenomena, which affect the particular categories. Those are briefly introduced and possible countermeasures are presented.

This approach contributes to maintain the balance of reactive power in operation and to reduce reactive power consumption of individual components as well as of entire transmission systems.

\section{References}

[1] J. Schlabbach, K. Rofalski, Power System Engineering, WILEY-VCH, Weinheim (2008),

[2] Wissenschaftlicher Ausschuss des VDE, Blindleistung, VDE-Verlag, Berlin (1963)

[3] G. Herold, Elektrische Energieversorgung I, J. Schlembach Fachverlag, Wildburgstetten (2005), 2nd Edition

[4] D.A. Deib, H.W. Hill, Optimal Firing-Angle Control of Cascaded HVDC converters for Minimum Reactive Power Demand, Applied Power Electronics Conference and Exposition, San Diego (1993)

[5] K. Heuck, K. Dettmann, D. Schulz, Elektrische Energieversogung, Vieweg + Teubner, Wiesbaden (2010), 8th Edition

[6] G. Herold, Elektrische Energieversorgung V, J. Schlembach Fachverlag, Wildburgstetten (2009)

[7] M. Davies et al., HVDC plus - Basics and Principle of Operation, http://www.energy.siemens.com/mx/pool/hq/powertransmission/HVDC/HVDC_Plus_Basics_and_Principle.pdf, accessed on 22th October 2012

[8] G. Herold, Elektrische Energieversorgung II, J. Schlembach Fachverlag, Wildburgstetten (2008), 2nd Edition

[9] G. Gemmell, J. Dorn, D. Retzmann, D. Soerangr, Prospects of Multilevel VSC Technologies for Power Transmission, IEEE Transmission and Distribution Conference and Exposition 2008, Chicago 